\title{
The effect of aerodynamic heat on the dynamics character of front body of assembling materials
}

\author{
LIU Chao ${ }^{1, a}$, LI Fan-chun ${ }^{1, b}$ \\ ${ }^{1}$ Transportation equipment and marine engineering, Dalian Maritime University, Dalian, 116026, \\ China \\ aemail: dmuhy@126.com, bemail:lee_fc@126.com
}

Keywords: Aero-engine; Temperature field; Thermal stress; Modal analysis; Multi-field coupling; Inherent frequency

\begin{abstract}
Aerodynamic heating would generate high temperature rise on aero engine structure and severe temperature gradient on the body of assembling materials at the front of gas inlet which would develop heterogeneous temperature and thermal stress field and influence the kinetic characteristics of mechanical structure. In this paper, a finite element model of Ceramic-SiC front body has been built up. The heterogeneous temperature field inside the front body was computed from fluid flow and thermal conduction. The thermal stress was achieved by thermo-solid coupling computation with the boundary condition of temperature field and mode analysis of prestress was carried out by loading thermo stress on the front body. Under the environment of liquid-thermal-solid multi-field coupling, through the investigation on the inherent frequency and the variation of vibration type of the front body at different temperatures, the results show that the thermo vibration frequency of Ceramic-SiC front body aroused by the aerodynamic heat could be approximately replaced by the inherent frequency of normal temperature below $500^{\circ} \mathrm{C}$, then a accelerated decrease of inherent frequency was observed when the temperature exceeded $600{ }^{\circ} \mathrm{C}$ and the decline rate reached the maximum value at $1050{ }^{\circ} \mathrm{C}$. Over $10 \%$ relative difference of first order inherent frequency was obtained. Therefore the effect of aerodynamic heat on the inherent frequency should be considered during the design process and a configuration should be made especially on the low order inherent frequency.
\end{abstract}

\section{Introduction}

With the modern aerocraft becoming faster and faster, aerodynamic heating problem tends to be more serious. Especially for the front body at the front of engine gas inlet, it has to bear high temperature which is caused by aerodynamic heating[1]. To consider the requirements of both strength and high temperature resistance, the researchers have designed a front body of assembling materials in recent years: use Ceramic to manufacture the forepart of the front body, allowing it to possess the performance of high temperature resistance and use silicon carbide to form the back body which can help to improve the strength of the front body. However, using multi-materials to make the front body brings a new problem: due to the differences of heat transfer coefficient, thermal expansion coefficient and elastic modulus of different materials, aerodynamic heating would not only generate high temperature on the front body, but also produce high temperature gradient, forming uneven temperature field and thermal stress field. This will affect the dynamic characteristics of the front body.

Actually the effect of aerodynamic heating on dynamic characteristics had been put forward by some scholars early in their studies on the wing flutter, and there are many literatures on aerodynamic heating vibration problems in recent years. Literature[2-4] proposed a fast algorithm which is suitable for solving heat flux distribution of aircraft surface in initial design, and it verified the practicability of the algorithm by comparing it with the test results of wind tunnel. Literature[5-7] expounded the thoughts and steps of thermal vibration analysis, and summarized the effect trend of aerodynamic heating on aircraft structure stiffness and vibration; in literature[8-9], Yang Bingyuan, Shi Xiaoming and other scholars not only elaborated a complete analysis process from aerodynamic heating to modal analysis in details, but also provided the mathematical model 
and solution method of thermo-elastic coupling and thermo-modal analysis. Literature[10-12] introduced and analyzed the calculation method of aerodynamic forces and aero-elastic problems in high speed unsteady regime, and proposed some issues of high speed aerocraft which need to be solved and paid attention to in the field of aeroelastics on the basis of previous studies.

The studies of above aerodynamic heating vibration problems are mostly against single material structure. There are not many thermal vibration studies on composite structure of assembling materials, and studies with engine front body of assembling materials as the research object are even fewer. In addition, the key steps and difficulties of thermal vibration calculation is the solution of heterogeneous temperature field. The traditional way is to treat the internal temperature of the structure as a uniform temperature field, set a constant temperature to act on the structure, conduct thermal stress calculation, and carry out kinetic analysis under the action of thermal stress. This method is simple and rapid, but it ignored the influence of temperature on material properties and the temperature gradient formed by the conduction of surface temperature. It also blurred the effects of aerodynamic heating on the vibration characteristics of the structure. Therefore this method has caused large error to the solution results of temperature field and inherent frequency. Against the shortcomings of the traditional study, a calculation model of Ceramic-SiC front body has been built in this paper to improve the calculation of temperature field, then based on multi-field coupling algorithm, the study on kinetic characteristics of composite front body under the action of aerodynamic heating is carried out by four steps (aerodynamic heating- temperature field analysis-thermal stress calculation -modal analysis).

\section{Calculation Principles}

During mathematical modeling, the calculation of heterogeneous temperature flied of internal front body can be divided into two parts: flow field analysis and solid heat conduction.In the region of flow field, aerodynamic model uses Favre average unsteady Navier-Stoke equation. In Cartesian coordinates, it is expressed in the form of vector equation:

$$
\frac{\partial}{\partial t}\left[\begin{array}{c}
\rho \\
\rho u_{1} \\
\rho u_{2} \\
\rho u_{3} \\
\rho E \\
\rho v_{1} \\
\rho v_{2}
\end{array}\right]+\frac{\partial}{\partial x_{i}}\left[\begin{array}{c}
\rho u_{i} \\
\rho u_{1} u_{i}+p \delta_{i 1} \\
\rho u_{2} u_{i}+p \delta_{i 2} \\
\rho u_{3} u_{i}+p \delta_{i 3} \\
\rho H u_{i} \\
\rho v_{1} u_{i} \\
\rho v_{2} u_{i}
\end{array}\right]-\frac{1}{R e} \cdot \frac{\partial}{\partial x_{i}}\left[\begin{array}{c}
0 \\
\tau_{i 1} \\
\tau_{i 2} \\
\tau_{i 3} \\
\tau_{i j} u_{j}-q_{i} \\
\varpi_{i}\left(v_{1}\right) \\
\varpi_{i}\left(v_{2}\right)
\end{array}\right]-\left[\begin{array}{c}
0 \\
0 \\
0 \\
0 \\
0 \\
s_{v_{1}} \\
s_{v_{2}}
\end{array}\right]=0
$$

In the formula, $\rho$ refers to density; P refers to pressure; $\mathrm{k}$ is turbulent kinetic energy; $\mathrm{E}$ and $\mathrm{H}$ are stagnation internal energy and stagnation enthalpy, respectively; $\tau_{i j}$ is the molecular Reynolds stress tensor; qi is the sum of molecular and turbulent heat vector; $v_{1} 、 v_{2}$ are two unknown variables which determine the viscosity $\left(\mu_{t}\right)$ of turbulence vortex; $s_{v_{m}}$ is the source item; Pr、Pr are the Prandtl number of laminar flow and turbulent flow, respectively; Re is the characteristic Reynolds number consisted of characteristic quantity which is introduced during the dimensionless.

When solving the temperature field, solid heat conduction calculation also need to be conducted in addition to flow field calculation: $\left(\frac{C_{t}}{\partial \tau}+K_{t}\right) T_{t}=P_{t}+\frac{K_{t}}{\partial \tau} T_{t-\Delta \tau}$

The thermal stress generalized Hooke's law is: $\sigma=\mathrm{D}\left(\varepsilon-\varepsilon_{T}\right)$

$$
\text { wherein: } \mathrm{D}=\frac{E}{2\left(1-v-2 v^{2}\right)}\left[\begin{array}{cccccc}
2(1-v) & 2 v & 2 v & 0 & 0 & 0 \\
2 v & 2(1-v) & 2 v & 0 & 0 & 0 \\
2 v & 2 v & 2(1-v) & 0 & 0 & 0 \\
0 & 0 & 0 & 1-2 v & 0 & 0 \\
0 & 0 & 0 & 0 & 1-2 v & 0 \\
0 & 0 & 0 & 0 & 0 & 1-2 v
\end{array}\right]
$$


In the formula, $\sigma$ is the elastic stress considering the influences of varying temperature, calling thermal stress; $\varepsilon$ the total strain; $\varepsilon_{T}$ is the thermal strain caused by temperature change. D refers to elastic matrix; E is the elastic modulus, and $v$ is Poisson's ratio.

$\sigma=\mathrm{D}\left(\varepsilon-\varepsilon_{T}\right)$, and obtain: $\varepsilon=\mathrm{S} \sigma+\varepsilon_{T}=\varepsilon_{\mathrm{E}}+\varepsilon_{T}$.

This article assumes that the mass matrix [M] of the front body is not affected by temperature; the temperature changing dynamic characteristics of the structure is mainly caused by the changes in stiffness matrix.

The temperature changes the elastic modulus of structure material, so the stiffness matrix is obtained and marked as $\left[K_{T}\right]$; the uneven change of structure temperature generates compressive thermal stresses in the object and changes the distribution of initial structural stiffness, thus the initial stress stiffness matrix is obtained and marked as $\left[K_{\sigma}\right]$. after taking full consideration of temperature effect, the structural stiffness matrix is marked as $[K]$. There is: $[K]=\left[K_{\sigma}\right]+\left[K_{T}\right]$.

Then, the thermal modal analysis equation of the structure considering the effects of temperature is: $\left(\left[K_{\sigma}\right]+\left[K_{T}\right]-\omega^{2}[M]\right)\{\varphi\}=0$ 。

\section{Simulation Calculation}

Fluent calculation can help to get the parameters of the front body at various states in the flow field. This paper takes temperature field as the major concern.

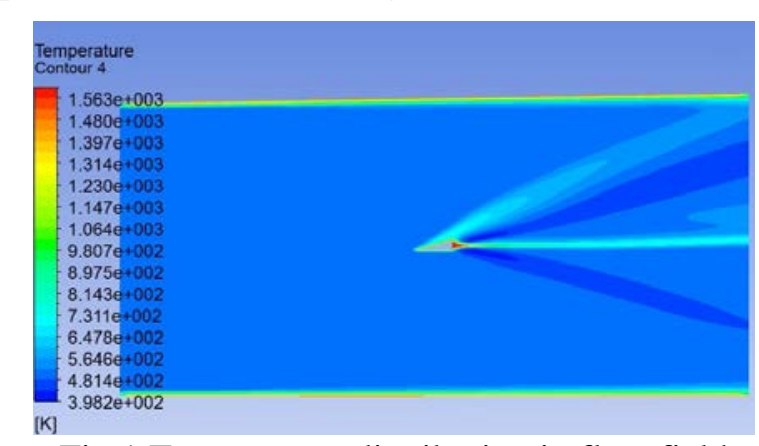

Fig.1 Temperature distribution in flow field

From figure 1 we can see that the flow field temperature of the front body tail is relatively high, as high as $1593 \mathrm{k}$. This will explain why the front body is required to possess high temperature resistant characteristics. In addition, we should notice that the result obtained by Fluent calculation only refers to the temperature of flow field around the front body, which cannot be directly used in the thermal stress calculation of the front body. As seen from figure 1, the internal temperature of the front body is not shown, so the flow field temperature must be turned to the internal temperature field of the front body.

In the same coordinate system, map the fluent temperature onto the front body surface, and then carry out heat conduction calculation. As two kinds of materials have different conduction coefficients, the calculated internal temperature field of the front body is an uneven temperature field.In order to know the distribution of temperature field in the whole front body, the paper used edge of body length 、 width 、 height for $\mathrm{X} 、 \mathrm{Y} 、 \mathrm{Z}$ axis to establish a new coordinate system, then to calculate the temperature distribution along the coordinate axis. 


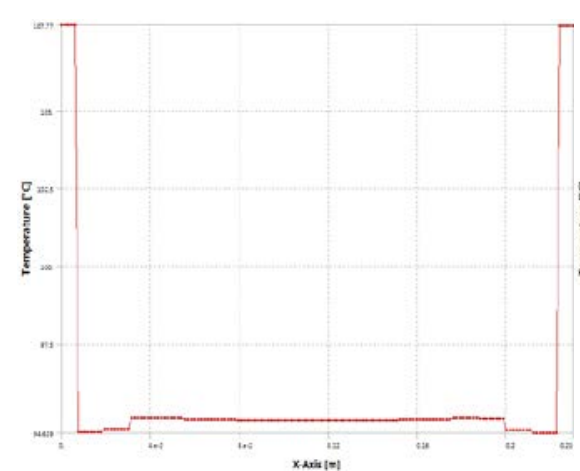

(a) along the $\mathrm{X}$ axis

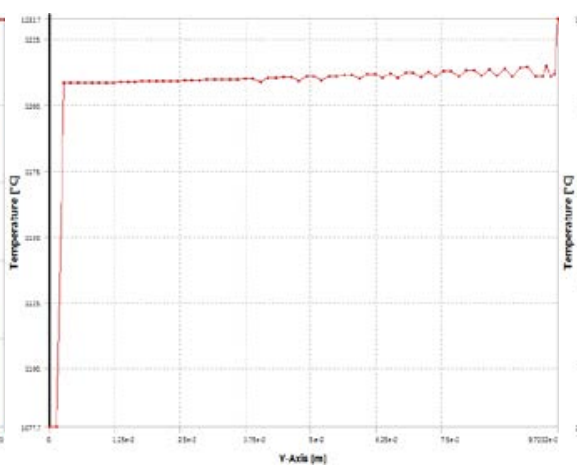

(b) along the $\mathrm{Y}$ axis

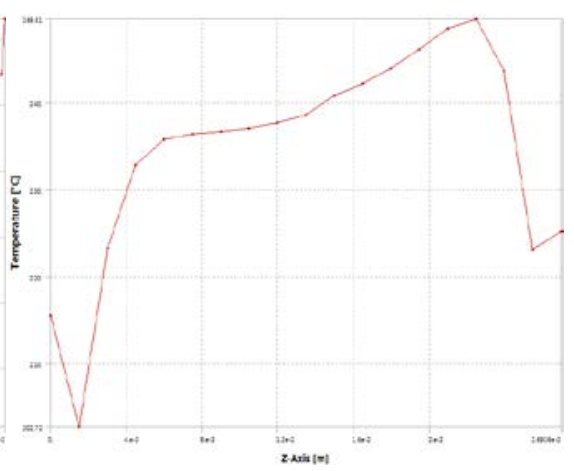

(c) along the $\mathrm{Z}$ axis

Fig.2 Temperature profile along the coordinate axis

Seen from (a) to (c) in figure 2, Temperature distribution along length direction is symmetrical, especially the temperature along the length direction is high at two ends and low in middle. Meanwhile, the temperature along the width direction is always rising, especially the front end temperature rises quickly. Furthermore, along the height direction, temperature varies irregularly.In a word, there are no significant distribution regularity in whole temperature field.

The research object of this article is a wedge-shaped front body, with Ceramic materials formed the forepart and silicon carbide materials formed the after body. The two parts are connected by mortise joint. Using adaptive method to divide it into hexahedral mesh, and 59582 grids are obtained in total. Take temperature field as thermal load and load it onto the front body to conduct heat - solid coupling calculation.

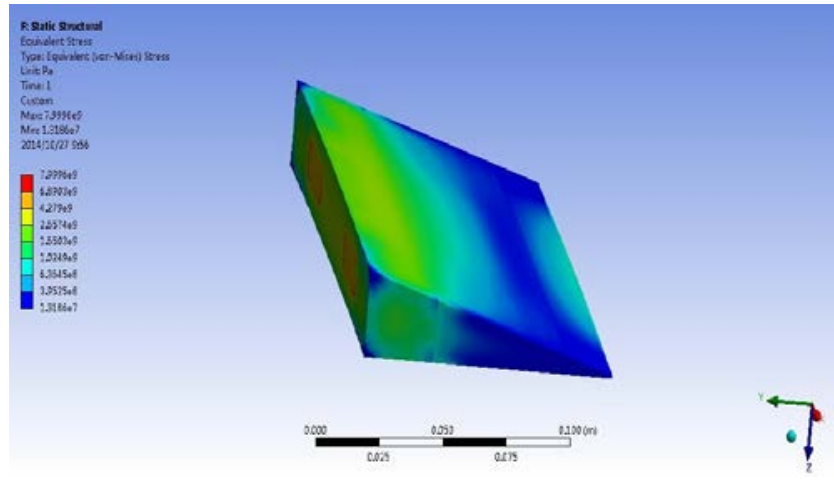

Fig.3 Thermal stress distribution

Seen from figure 3, distribution of thermal stress is heterogeneous. It mainly concentrated on the backend of the front body, and is smaller at the forepart. So in the design of high performance front body, different materials are adopted; high strength materials are usually chosen for the back-end.

\section{Modal analysis}

Divide the aerodynamic heat temperature of launched stable phase into ten evenly spaced temperature points, and successively calculate the first eight orders' inherent frequency of the front body at each temperature point. The results are shown in table 1.

Table1. Inherent frequency at different temperature

\begin{tabular}{ccccccccc}
\hline \multirow{2}{*}{$\begin{array}{c}\text { Temperature } \\
/{ }^{\circ} \mathrm{C}\end{array}$} & \multicolumn{8}{c}{ Inherent frequency $/ \mathrm{Hz}$} \\
\cline { 2 - 9 } & $1^{\text {st }}$ order & $2^{\text {nd }}$ order & $3^{\text {rd }}$ order & $4^{\text {th }}$ order & $5^{\text {th }}$ order & $6^{\text {th }}$ order & $7^{\text {th }}$ order & $8^{\text {th }}$ order \\
\hline 132 & 1978.1 & 2475.6 & 3420.4 & 4935.3 & 5882.5 & 6508.4 & 6674.6 & 7465.8 \\
264 & 1972.9 & 2468.6 & 3408.9 & 4923.3 & 5871.5 & 6496.4 & 6671.5 & 7456.5 \\
396 & 1964.3 & 2458.3 & 3392.5 & 4904.8 & 5854.8 & 6477.3 & 6663.8 & 7440.5 \\
528 & 1949.6 & 2441.7 & 3367.6 & 4875.8 & 5827.4 & 6446.6 & 6649.9 & 7413.8 \\
660 & 1938.4 & 2427.5 & 3344.5 & 4850.4 & 5806.1 & 6419.3 & 6643.2 & 7393.3 \\
792 & 1920.5 & 2408.6 & 3317.1 & 4819.2 & 5776.4 & 6378.9 & 6628.6 & 7359.9
\end{tabular}




\begin{tabular}{ccccccccc}
924 & 1887.3 & 2372.5 & 3264.9 & 4757.6 & 5719.9 & 6312.8 & 6599.1 & 7301.3 \\
1056 & 1853.1 & 2335.2 & 3209.1 & 4689.4 & 5655.3 & 6241.2 & 6564.5 & 7231.2 \\
1188 & 1812.9 & 2291.6 & 3145.7 & 4610.5 & 5581.5 & 6154.3 & 6523.4 & 7150.1 \\
1320 & 1779.1 & 2257.3 & 3101.6 & 4554.1 & 5528.6 & 6078.7 & 6495.1 & 7087.9 \\
\hline
\end{tabular}

It can be found in calculation that when temperature changes, the inherent frequency of same order vibration will also change, but vibration mode has no significant alteration. Namely: aerodynamic heat would change the inherent frequency of the front body, but has little influence on vibration mode. So this paper mainly focuses on the changing law of inherent frequency with the temperature.
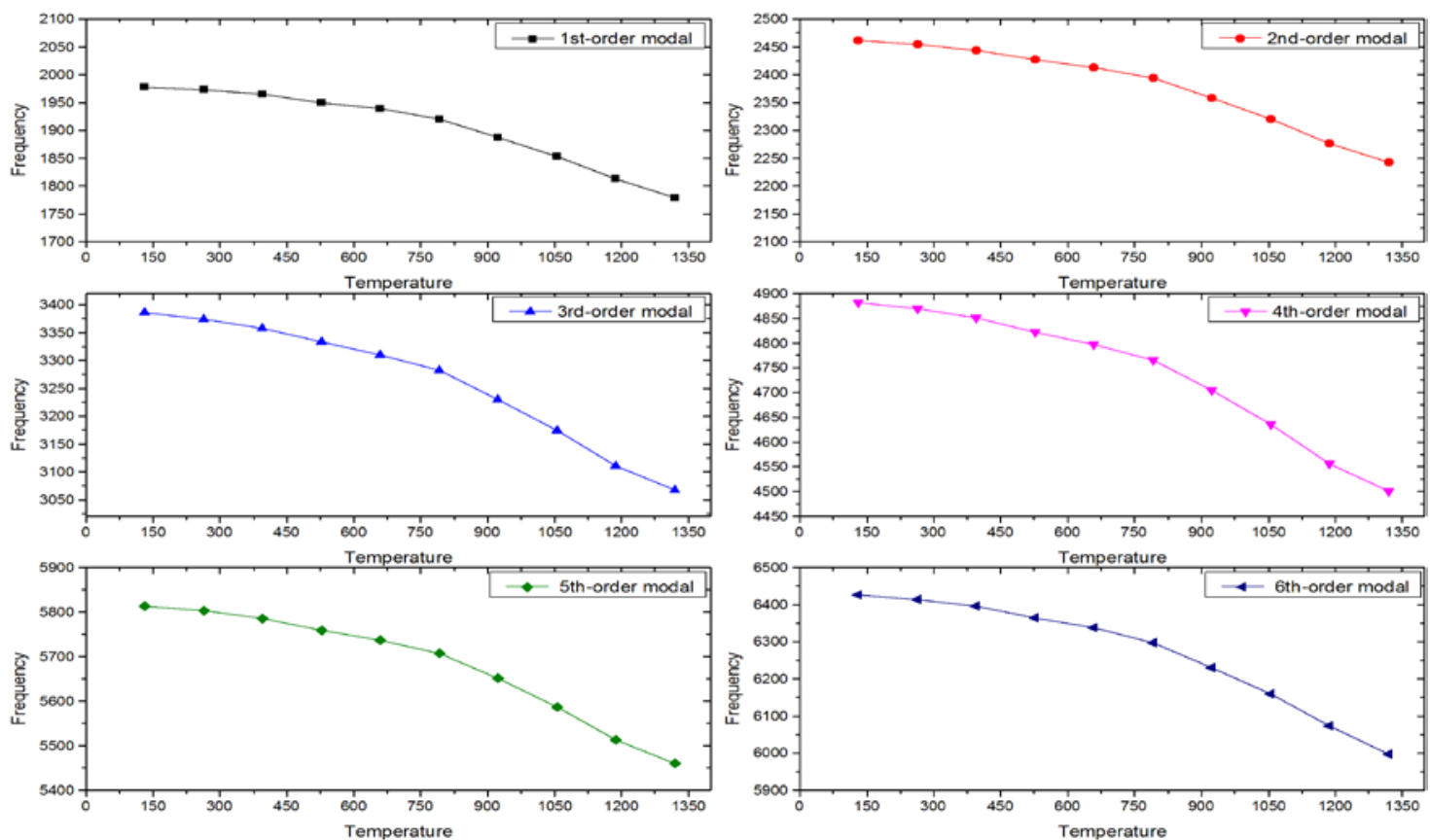

Fig.4 The relations of frequency and temperature

Seen from figure 4, the Inherent frequencies of each order drops with the increase of temperature, making the external interference more easily to cause the resonance of the front body. So the influence of temperature rise caused by aerodyne- mic heating on the inherent frequency of the front body need to be considered in design process.
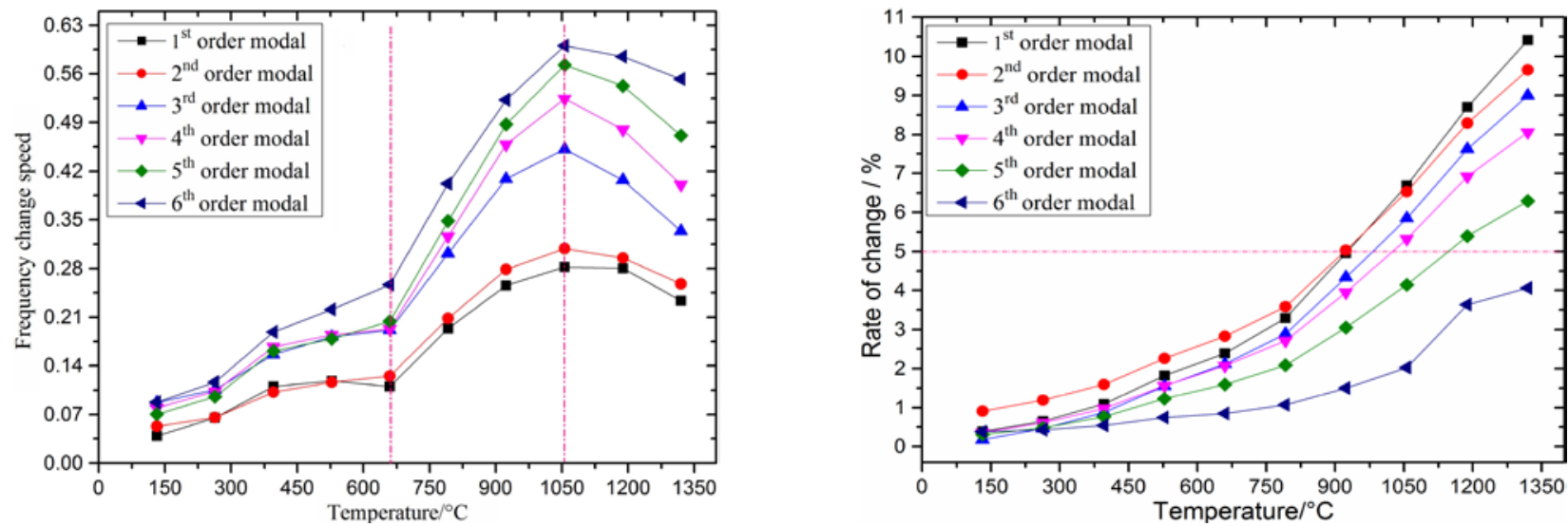

Fig.5 Frequency change speed and temperature Fig.6 Frequency change rate and temperature

Although it is already known that temperature rise leads to the decrease of inherent frequency of the front body, the frequency declining rate of each order is different. Meanwhile, the frequency changing rate is also different in the process of aerodynamic heating. As can be seen from figure 5, the frequency declining speed at the early stage of aerodynamic heating reduces at a lower rate, and the frequency declining rate of each order is not regular. When temperature exceeds $650{ }^{\circ} \mathrm{C}$, the 
declining speed of inherent frequency significantly accelerates; the inherent frequency declining rate of high order is bigger than that of low order. At the end of heating process, with the gradual balance of temperature field and thermal stress of the structure, the frequency declining rate of each order starts to decrease obviously.

Meanwhile, as can be seen from figure 6, the frequency changing rate is relatively small at low temperature, and the thermo-vibration frequency could be approximately replaced by the inherent frequency of normal temperature; with the rise of temperature, frequency changing rate increases, and the changing rate of low order is bigger than that of high order. We can see the frequency changing rate of order 6 is lower than 5\%, which means the frequency changing rate of higher order is even lower. So in design, only the influence of aerodynamic heat on low order inherent frequency needs to be taking into consideration.

\section{Conclusions}

Through seamless connection and invocation of data under multi-field coupling environment of liquid-solid-thermal structure, this article has carried out more accurate thermal stress and modal analyses on the front body of assembling materials. And the following conclusions are obtained:

The thermal stress generated by the action of aerodynamic heat on the front body mainly concentrated on the backend and is smaller at the forepart. Therefore it is necessary to adopt high strength materials for the tail of the front body.

The frequency declining speed at the early stage of aerodynamic heating reduces at a lower rate, and the frequency declining rate of each order is not regular. When temperature exceeds $650{ }^{\circ} \mathrm{C}$, the declining speed of inherent frequency significantly accelerates; the inherent frequency declining rate of high order is bigger than that of low order. Declining rate reached the maximum value at $1050{ }^{\circ} \mathrm{C}$. At the end of heating process, with the gradual balance of temperature field and thermal stress of the structure, the frequency declining rate of each order starts to decrease obviously.

The thermal vibration frequency of the front body aroused by the aerodynamic heat could be approximately replaced by the inherent frequency of normal temperature below $500^{\circ} \mathrm{C}$; when the temperature exceed $1100{ }^{\circ} \mathrm{C}$, relative difference of the first four orders' inherent frequencies can exceed 5\%. Especially for the first order vibration, the biggest difference can reach up to $10 \%$. This is why a configuration should be made especially on the low order inherent frequency during design process.

\section{References}

[1] SUN Yang, LU Jian, ZHENG Yan. Temperature distribution and thermal stress analysis for turbojet engine turbine guide blade[J].Journal of propulsion technology. 2004, 25(4).357-359

[2] Lv Li-li, Zhang Wei-wei, Ye Zheng-yin. Predicting Heating Distributions for Hypersonic Reentry Bodies[J]. Chinese Journal of Applied Mechanics. 1995, 23(2). 259-262

[3] Kang Hong-lin,Yan Chao.Numerical study of aeroheating predictions for hypersonic reentry bodies[J]. Journal of Beijing University of Aeronautics and Astronautics. 2006,32(12). 1395-1398

[4] LI Hui-ping, DONG Wei.Calculation of Aerodynamic Heating Distributions for Hypersonic Reentry Blunt Nosed Bodies[J]. Aerospace Shanghai. 2010, 27(2):18-22

[5] CHENG Wen-jun. The effect of aerodynamic heat on aeroelasticity of hypersonic vehicles[J]. Modern defence technology.1998(3):21-28.

[6] Andrew M B.Temperature-dependent modal test analysis correla tion of X-34 fastrac composite rocket nozzle [R]. AIAA,2000

[7] Haider N.Arafat, Ali H.Nayfeh. Nonlinear Interactions in the Responses of Heated Annular Plates[R]. AIAA, 2004

[8] SHI Xiao-ming, YANG Bing-yuan.Temperature Field and Mode Analysis of Flat Plate with 
Thermal Environment of Transient Heating[J]. Computer Aided Engineering.2006, 15(S1): 15-18

[9] YANG Bing-yuan, SHI Xiao-ming, LIANG Qiang. Investigation and development of the multi-physics coupling dynamics on the hypersonic winged missiles [J].CARS-2007

[10]Yang Chao,Xu Yun,Xie Chang-chuan.Review of studies on aeroelasticity of hypersonic vehicle[J]. Acta Aeronautica Et Astronautica Sinica.2010,31(01).1-11

[11]Li Guo-shu, Wan Zhi-qiang, Yang Chao. Integrated analysis of aerothermal-aeroelastic wings in hypersonic flow[J].Journal of Beijing University of Aeronautics and Astronautics. 2012,38(01):53-58

[12]YANG Qiong-liang, SHI Xiao-ming, TANG Guo-an.Flutter analysis for a high-aspect ratio wing with finite element method[J].JOURNAL OF VIBRATION AND SHOCK.2011.30(5): 73-75 